\title{
General existence of minimal surfaces of genus zero with catenoidal ends and prescribed flux
}

\author{
Shin Kato, Masaaki Umehara and Kotaro Yamada
}

\section{Introduction.}

Let $x: \mathbf{C} \cup\{\infty\} \backslash\left\{q_{1}, \ldots, q_{n}\right\} \rightarrow \mathbf{R}^{3}$ be a complete conformal minimal immersion. For each end $q_{j}(j=1, \ldots, n)$ of $x$, the flux vector is defined by

$$
\varphi_{j}:=\int_{\gamma_{j}} \vec{n} d s
$$

where $\gamma_{j}$ is a positively oriented curve surrounding $q_{j}$, and $\vec{n}$ is the conormal such that $\left(\gamma_{j}^{\prime}, \vec{n}\right)$ is positively oriented. It is well known that the flux vectors satisfy a "balancing" condition so called the flux formula

$$
\sum_{j=1}^{n} \varphi_{j}=0
$$

The minimal immersion $x$ is called an $n$-end catenoid if each end $q_{j}$ is of catenoid type. The catenoid and the Jorge-Meeks surfaces [JM] are typical ones. Recently, new examples of $n$-end catenoids have been found by [Kar], [L], [Xu], [Ross1], [Ross2], [Kat] and [UY]. For any $n$-end catenoid $x$, each flux vector $\varphi_{j}$ is proportional to the limit normal vector $\nu\left(q_{j}\right)$ with respect to the end $q_{j}$, and the scalar $w\left(q_{j}\right):=\varphi_{j} / 4 \pi \nu\left(q_{j}\right)$ is called the weight of the end $q_{j}$. In this case, the flux formula can be rewritten as follows:

$$
\sum_{j=1}^{n} 4 \pi w\left(q_{j}\right) \nu\left(q_{j}\right)=0 .
$$

It should be remarked that $w\left(q_{j}\right)$ may take a negative value.

We consider the inverse problem of the flux formula proposed in [Kat] and [KUY1] as follows: 
Problem. For given unit vectors $v:=\left\{v_{1}, \ldots, v_{n}\right\}$ in $\mathbf{R}^{3}$, and nonzero real numbers $a:=\left\{a^{1}, \ldots, a^{n}\right\}$ satisfying $\sum_{j=1}^{n} a^{j} v_{j}=0$ (we call such a pair $(v, a)$ flux data), is there a (non-branched) n-end catenoid $x: \mathbf{C} \cup\{\infty\} \backslash$ $\left\{q_{1}, \ldots, q_{n}\right\} \rightarrow \mathbf{R}^{3}$ such that $\nu\left(q_{j}\right)=v_{j}$ and $a_{j}$ is the weight at the end $q_{j}$ ?

We remark that Kusner also proposed a similar question (see [Ross1]). Rosenberg and Toubiana [RT] found solutions with branch points in the class that the Gauss map is of degree 1. But if one wishes a non-branched solution, the degree of its Gauss map must be $n-1$, which is the case just treated in this paper.

The problem is not exactly affirmative. By the classification of Lopez [L], we can see that the answer for $n \leq 3$ is "Yes" except for the case when two of $\left\{v_{j}\right\}_{j=1}^{n}$ coincide. Moreover, for $n \geq 4$, some obstructions exist as closed conditions in the space of flux data as shown in our previous paper [KUY1]. In spite of these obstructions, the authors also showed in [KUY1] that the inverse problem is true for almost all flux data $(v, a)$ when $n=4$. In this paper, we treat the case $n \geq 5$ and show the following theorem:

Theorem. For each integer $n \geq 3$, the problem is solved for almost all flux data.

In Section 1, we reduce the inverse problem to seeking a sampling point satisfying certain non-degeneracy conditions. Two lemmas in Appendix A are applied to complete the reduction. In Section 2, we shall give a proof of Theorem. However, required technical calculations are done in Section 3 and Appendix B.

The above general existence theorem does not apply for the case that all flux vectors lie in the same plane, since such flux data are contained in a measure zero subset in the set of all flux data. We say that such minimal surfaces are of Type II. In [KUY2], we show that our approach in this paper can be modified even for such a specified case and prove the general existence of $n$-end catenoids $(n \leq 8)$ of Type II. Recently, KusnerSchmitt [KS] explain the moduli space of minimal surfaces with embedded planar ends by using the term of spin structure of Riemann surfaces. It should be remarked that our approach can also be interpreted in terms of spin structure (see Remark 1.5).

The authors are very grateful to Professors Yusuke Sakane, Ichiro Enoki and Koji Cho for valuable discussions and encouragement. 


\section{Reduction.}

The flux vector $\varphi_{j}(j=1, \ldots, n)$ given by $(0.1)$ in Introduction can be rewritten as follows:

$$
\varphi_{j}:=-\operatorname{Im}\left(\oint_{\gamma_{j}}\left(1-g^{2}\right) \omega, \oint_{\gamma_{j}} \sqrt{-1}\left(1+g^{2}\right) \omega, \oint_{\gamma_{j}} 2 g \omega\right),
$$

where $(g, \omega)$ is the Weierstrass data of the minimal immersion

$$
x: \mathbf{C} \cup\{\infty\} \backslash\left\{q_{1}, \ldots, q_{n}\right\} \rightarrow \mathbf{R}^{3}
$$

given by

$$
g:=\frac{\partial x^{3}}{\partial x^{1}-\sqrt{-1} \partial x^{2}}, \quad \omega:=\partial x^{1}-\sqrt{-1} \partial x^{2} .
$$

On the other hand, the well-known Weierstrass representation is written as

$$
x=\operatorname{Re}\left(\int_{z_{0}}^{z}\left(1-g^{2}\right) \omega, \int_{z_{0}}^{z} \sqrt{-1}\left(1+g^{2}\right) \omega, \int_{z_{0}}^{z} 2 g \omega\right) .
$$

In particular, the monodromy vector of the immersion around the end $q_{j}$ (resp. the flux vector of $q_{j}$ ) is the real part (resp. the imaginary part) of the residue of the holomorphic vector

$$
\partial x=\frac{1}{2}\left(\left(1-g^{2}\right) \omega, \sqrt{-1}\left(1+g^{2}\right) \omega, 2 g \omega\right),
$$

around the end $z=q_{j}$. We have shown in the previous paper [KUY1] that the inverse problem of the flux formula reduces to finding solutions of a system of algebraic equations:

Theorem 1.1 ([KUY1]). Let $(v, a)$ be a pair of unit vectors

$$
v=\left\{v_{1}, \ldots, v_{n}\right\}
$$

in $\mathbf{R}^{3}$ and nonzero real numbers $a=\left\{a^{1}, \ldots, a^{n}\right\}$ satisfying the balancing condition:

$$
\sum_{j=1}^{n} a^{j} v_{j}=0
$$


Then there is an (evenly) branched $n$-end catenoid

$$
x: \mathbf{C} \cup\{\infty\} \backslash\left\{q_{1}, \ldots, q_{n}\right\} \rightarrow \mathbf{R}^{3} \quad\left(q_{j} \neq \infty\right)
$$

such that the induced metric is complete at the end $q_{j}, \nu\left(q_{j}\right)=v_{j}$ and $a^{j}$ is the weight at the end $q_{j}(j=1, \ldots, n)$, if and only if there exist complex numbers $b^{1}, \ldots, b^{n}$ satisfying the following conditions:

$$
\left\{\begin{array}{l}
b^{j} \sum_{\substack{k=1 \\
k \neq j}}^{n} b^{k} \frac{p_{j}-p_{k}}{q_{j}-q_{k}}=a^{j} \\
b^{j} \sum_{\substack{k=1 \\
k \neq j}}^{n} b^{k} \frac{\overline{p_{j}} p_{k}+1}{q_{j}-q_{k}}=0
\end{array} \quad(j=1, \ldots, n)\right.
$$

where $p_{j}:=\sigma\left(v_{j}\right), \sigma: S^{2} \rightarrow \mathbf{C} \cup\{\infty\}$ is the stereographic projection, and we assume $p_{j} \neq \infty$.

Moreover, the surface $x$ has no branch points if and only if the polynomials

$$
\begin{aligned}
& Q(z):=\sum_{j=1}^{n} b^{j} \prod_{\substack{k=1 \\
k \neq j}}^{n}\left(z-q_{k}\right), \\
& P(z):=\sum_{j=1}^{n} p_{j} b^{j} \prod_{\substack{k=1 \\
k \neq j}}^{n}\left(z-q_{k}\right)
\end{aligned}
$$

are mutually prime and one of them has degree $n-1$.

Remark 1.2. When $p_{j}=r q_{j}$, the theorem reduces to the results in the first author [Kat]. In this case the system (1.3) and (1.4) reduces to

$$
\left\{\begin{array}{l}
r b^{j} \sum_{\substack{k=1 \\
k \neq j}}^{n} b^{k}=a^{j} \\
b^{j} \sum_{\substack{k=1 \\
k \neq j}}^{n} b^{k} \frac{|r|^{2} \overline{q_{j}} q_{k}+1}{q_{j}-q_{k}}=0
\end{array} \quad(j=1, \ldots, n) .\right.
$$

As seen in [Kat], the surface has no branch point if and only if $\beta$ := $\sum_{j=1}^{n} b^{j} \neq 0$. By using the relation

$$
\frac{P(z)}{Q(z)}=r z-\frac{r \beta}{\sum_{j=1}^{n} \frac{b^{j}}{z-q_{j}}}
$$


it is also checked directly from the last condition of the theorem.

Remark 1.3. The position of the ends $\left\{q_{1}, \ldots, q_{n}\right\}$ in the source domain $\mathbf{C} \cup\{\infty\}$ has the freedom of Möbius transformations. For example, the following normalization is possible:

$$
q_{1}=1, \quad q_{n-1}+q_{n-2}=0, \quad q_{n}=0 .
$$

Remark 1.4. The system of the equations (1.3) and (1.4) has another expression

$$
\left\{\begin{array}{l}
b^{j} \sum_{\substack{k=1 \\
k \neq j}}^{n} b^{k} \frac{1}{q_{j}-q_{k}}=a^{j} \frac{\overline{p_{j}}}{\left|p_{j}\right|^{2}+1}, \\
b^{j} \sum_{\substack{k=1 \\
k \neq j}}^{n} b^{k} \frac{p_{j}+p_{k}}{q_{j}-q_{k}}=a^{j} \frac{\left|p_{j}\right|^{2}-1}{\left|p_{j}\right|^{2}+1} .
\end{array}\right.
$$

Moreover we may replace (1.7) by

$$
p_{j} b^{j} \sum_{\substack{k=1 \\ k \neq j}}^{n} b^{k} \frac{p_{k}}{q_{j}-q_{k}}=-a^{j} \frac{p_{j}}{\left|p_{j}\right|^{2}+1} .
$$

In fact, if we set

$$
\gamma_{j}:=b^{j} \sum_{\substack{k=1 \\ k \neq j}}^{n} b^{k} \frac{1}{q_{j}-q_{k}}, \quad \delta_{j}:=b^{j} \sum_{\substack{k=1 \\ k \neq j}}^{n} b^{k} \frac{p_{k}}{q_{j}-q_{k}} \quad(j=1, \ldots, n),
$$

then (1.3) and (1.4) are written as

$$
p_{j} \gamma_{j}-\delta_{j}=a^{j}, \quad \gamma_{j}+\overline{p_{j}} \delta_{j}=0 .
$$

It is equivalent to the relations

$$
\gamma_{j}=a^{j} \frac{\overline{p_{j}}}{\left|p_{j}\right|^{2}+1}, \quad p_{j} \gamma_{j}+\delta_{j}=a^{j} \frac{\left|p_{j}\right|^{2}-1}{\left|p_{j}\right|^{2}+1},
$$

that is (1.7) and (1.8). On the other hand,

$$
p_{j} \gamma_{j}=a^{j} \frac{\left|p_{j}\right|^{2}}{\left|p_{j}\right|^{2}+1}=a^{j} \frac{\left|p_{j}\right|^{2}-1}{\left|p_{j}\right|^{2}+1}+\frac{a^{j}}{\left|p_{j}\right|^{2}+1}=p_{j} \gamma_{j}+\delta_{j}+\frac{a^{j}}{\left|p_{j}\right|^{2}+1}
$$

which yields (1.9). 
Remark 1.5. The construction of $n$-end catenoids mentioned above is related to the spinor representation of minimal surfaces (cf. [KS]);

$$
x=\operatorname{Re}\left(\int_{z_{0}}^{z}\left(s_{1}{ }^{2}-s_{2}^{2}\right), \int_{z_{0}}^{z} \sqrt{-1}\left(s_{1}{ }^{2}+s_{2}^{2}\right), \int_{z_{0}}^{z} 2 s_{1} s_{2}\right),
$$

where $\left(s_{1}, s_{2}\right)$ is a pair of meromorphic sections of the half-canonical bundle on $\mathbf{C} \cup\{\infty\}$. In fact, $s_{1}$ and $s_{2}$ have the following explicit expressions in this case:

$$
s_{1}:=\frac{Q(z)}{R(z)} \sqrt{-d z}, \quad s_{2}:=\frac{P(z)}{R(z)} \sqrt{-d z}
$$

where we set

$$
R(z):=\prod_{k=1}^{n}\left(z-q_{k}\right)
$$

Theorem 1.1 produces many $n$-end catenoids as seen in [Kat] and [KUY1]. First, we fix our attention to the equation (1.4). We consider a matrix

$$
A_{p}:=\left(\frac{\overline{p_{j}} p_{k}+1}{q_{j}-q_{k}}\right)_{j, k=1, \ldots, n},
$$

where the diagonal components are interpreted as 0 . Then the vector ${ }^{t}\left(b^{1}, \ldots, b^{n}\right)$ belongs to the kernel of the matrix $A_{p}$. As shown in the later sections, it is reasonable to expect that the rank of the matrix $A_{p}$ is generically $n-1$. In this case, ${ }^{t}\left(b^{1}, \ldots, b^{n}\right)$ should be proportional to any column vector of the cofactor matrix $\widetilde{A}_{p}$ of $A_{p}$. (By the definition, $A_{p} \widetilde{A}_{p}=\widetilde{A}_{p} A_{p}=\left(\operatorname{det} A_{p}\right) I$ holds. $)$ So we set

$b_{p}(q)={ }^{t}\left(b_{p}^{1}(q), \ldots, b_{p}^{n}(q)\right):=$ the $n$-th column of the cofactor matrix $\widetilde{A}_{p}(q)$.

Now we reformulate the problem: For fixed $p:=\left(p_{1}, \ldots, p_{n}\right) \in \mathbb{C}^{n}$, define a rational map between two complex projective spaces

$$
\mathcal{F} \ell_{p}=\left[f_{p}^{1}, \ldots, f_{p}^{n}\right]: \mathbf{P}^{n-1} \rightarrow \mathbf{P}^{n-1}
$$

by

$$
f_{p}^{j}\left(q_{1}, \ldots, q_{n}\right):=b_{p}^{j}(q) \sum_{k \neq j} b_{p}^{k}(q) \frac{p_{j}-p_{k}}{q_{j}-q_{k}} \quad(j=1, \ldots, n) .
$$


We set

$$
f \ell_{p}^{j}(q):=\Delta(q)^{5} \cdot f_{p}^{j}(q)
$$

where $\Delta(q)$ is the difference product defined by

$$
\Delta\left(q_{1}, \ldots, q_{n}\right):=\prod_{j>k}^{n}\left(q_{j}-q_{k}\right)
$$

It is easily seen that each $f \ell_{p}^{j}$ is a homogeneous polynomial in $q_{1}, \ldots, q_{n}$ and $\mathcal{F} \ell_{p}$ has another expression

$$
\mathcal{F} \ell_{p}=\left[f \ell_{p}^{1}, \ldots, f \ell_{p}^{n}\right]
$$

This new formulation is reasonable in the following two senses:

- Any homothety of $n$-end catenoids changes their weights $\left(a^{1}, \ldots, a^{n}\right)$ only by a constant multiplication. It allows us to projectify the image of $\mathcal{F} \ell_{p}$.

- Changing coordinates of $n$-end catenoids by homothetic transformations corresponds to complex multiplications of $\left(q_{1}, \ldots, q_{n}\right)$ (see Remark 1.3). It allows us to projectify the domain of $\mathcal{F} \ell_{p}$.

Since $p_{j}$ is the stereographic image of $v_{j}$, the balancing condition (1.2) is rewritten as

$$
\sum_{j=1}^{n} \frac{\left|p_{j}\right|^{2}-1}{\left|p_{j}\right|^{2}+1} a^{j}=0, \quad \sum_{j=1}^{n} \frac{\overline{p_{j}}}{\left|p_{j}\right|^{2}+1} a^{j}=0 .
$$

We define a subspace $\mathcal{W}_{p}^{n-4}$ in $\mathbf{P}^{n-1}$ by

$$
\begin{aligned}
\mathcal{W}_{p}^{n-4}:= & \left\{\left[a^{1}, \ldots, a^{n}\right] \in \mathbf{P}^{n-1} ;\right. \\
& \left.\sum_{j=1}^{n} \frac{\left|p_{j}\right|^{2}-1}{\left|p_{j}\right|^{2}+1} a^{j}=0, \sum_{j=1}^{n} \frac{\overline{p_{j}}}{\left|p_{j}\right|^{2}+1} a^{j}=0, \sum_{j=1}^{n} \frac{p_{j}}{\left|p_{j}\right|^{2}+1} a^{j}=0\right\} .
\end{aligned}
$$

We will show that for open dense $p \in \mathbf{C}^{n}$, the image of the map $\mathcal{F} \ell_{p}$ is open dense in $\mathcal{W}_{p}^{n-4}$, and next show that it covers open dense subset of the totally real set

$$
\mathcal{W}_{\mathbf{R}}=\left\{[a] \in \mathcal{W}_{p}^{n-4} ; a^{j} \in \mathbb{R},(j=1, \cdots, n)\right\}
$$


Then the image of the map $\mathcal{F} \ell_{p}$ contains $[a] \in \mathcal{W}_{\mathbf{R}}$ for almost all flux data $(p, a)$, and Theorem in Introduction is obtained. If $\mathcal{F} \ell_{p}$ is a holomorphic map and there is a point at which the rank of $d \mathcal{F} \ell_{p}$ is $n-4$, the surjectivity of the map follows by the proper mapping theorem (see [GR]). But unfortunately, the map $\mathcal{F} \ell_{p}$ is singular on the set $\bigcap_{j=1}^{n} \mathrm{Z}\left(f \ell_{p}^{j}\right)$, where $\mathrm{Z}\left(f \ell_{p}^{j}\right)$ is the set of zeros of $f \ell_{p}^{j}$. As shown below, we will overcome this difficulty by a usual blowing up process.

From here, assume $\operatorname{dim}\left\langle v_{1}, \ldots, v_{n}\right\rangle=3$, where $v_{j}:=\sigma^{-1}\left(p_{j}\right)$ and $\sigma$ is the stereographic projection. Then clearly $\operatorname{dim} \mathcal{W}_{p}^{n-4}=n-4$. This implies that $\operatorname{dim} \mathcal{W}_{p}^{n-4}=n-4$ holds for open dense $p \in \mathbf{C}^{n}$. Now we have the following lemma:

Lemma 1.6. For each $p \in \mathbf{C}^{n}$, the following relation holds:

$$
\mathcal{F} \ell_{p}\left(\mathrm{Z}\left(\lambda_{p}\right) \backslash \bigcap_{j=1}^{n} \mathrm{Z}\left(f \ell_{p}^{j}\right)\right) \subset \mathcal{W}_{p}^{n-4}
$$

where $\lambda_{p}$ is the determinant of the matrix $\Delta \cdot A_{p}$ and $\mathrm{Z}\left(\lambda_{p}\right)$ is the set of zeros of the homogeneous polynomial $\lambda_{p}$.

Proof. Let $q \in \mathrm{Z}\left(\lambda_{p}\right) \backslash \bigcap_{j=1}^{n} \mathrm{Z}\left(f \ell_{p}^{j}\right)$. If $\Delta(q)=0$, then it is easy to see that $q \in \bigcap_{j=1}^{n} \mathrm{Z}\left(f \ell_{p}^{j}\right)$. Hence $\Delta(q) \neq 0$, and we get (1.3) with $b^{j}=b^{j}(q)(j=$ $1, \ldots, n)$. Recall Remark 1.4. Then the assertion of the lemma immediately follows by summing up (1.8), (1.7) and (1.9) for $j=1, \ldots, n$.

We define an $(n-1)$-matrix $J_{p}$ by

$$
J_{p}:=\left(\left(f_{p}^{n}\right)^{2}\left\{\frac{\partial \operatorname{det} A_{p}}{\partial q_{n}} \cdot \frac{\partial \stackrel{\circ}{f_{p}^{k}}}{\partial q_{j}}-\frac{\partial \operatorname{det} A_{p}}{\partial q_{j}} \cdot \frac{\partial \stackrel{\circ}{f_{p}^{k}}}{\partial q_{n}}\right\}\right)_{k, j=1, \ldots, n-1}
$$

where

$$
\stackrel{\circ}{f_{p}^{j}}:=\frac{f_{p}^{j}}{f_{p}^{n}} \quad(j=1, \ldots, n-1) .
$$

The matrix $J_{p}$ has a direct expression

$$
\begin{aligned}
& J_{p}= \\
& \left(\frac{\partial \operatorname{det} A_{p}}{\partial q_{n}}\left\{\frac{\partial f_{p}^{k}}{\partial q_{j}} f_{p}^{n}-f_{p}^{k} \frac{\partial f_{p}^{n}}{\partial q_{j}}\right\}-\frac{\partial \operatorname{det} A_{p}}{\partial q_{j}}\left\{\frac{\partial f_{p}^{k}}{\partial q_{n}} f_{p}^{n}-f_{p}^{k} \frac{\partial f_{p}^{n}}{\partial q_{n}}\right\}\right)_{k, j=1, \ldots, n-1} .
\end{aligned}
$$


The following proposition plays an important role to prove Theorem in Introduction.

Proposition 1.7. Suppose that there exist $u_{0} \in \mathbf{C}^{n}$ and a point $c=$ $\left[c_{1}, \ldots, c_{n}\right] \in \mathbf{P}^{n-1}$ satisfying the following conditions:

(1) $c_{1}, \ldots, c_{n}$ are all distinct;

(2) The rank of the matrix $A_{u_{0}}(c)$ is $n-1$;

(3) $\partial \operatorname{det} A_{u_{0}} / \partial q_{n}$ does not vanish at $q=c$;

(4) The rank of the matrix $J_{u_{0}}(c)$ is $n-4$;

(5) Two polynomials $P(z)$ and $Q(z)$ defined in (1.6) and (1.5) associated with the data $(q, p)=\left(c, u_{0}\right)$ and $b=b_{u_{0}}(c)$ are mutually prime and one of them has degree $n-1$;

(6) $f_{u_{0}}^{j}(c) \neq 0(j=1, \ldots, n)$;

(7) $c_{j} \neq 0(j=1, \ldots, n-1)$.

Then there exists an open dense subset $U \subset \mathbf{C}^{n}$ and an open dense subset $\Omega_{p}$ of the totally real set $\mathcal{W}_{\mathbf{R}}=\left\{[a] \in \mathcal{W}_{p}^{n-4} ; a_{j} \in \mathbf{R}\right\}$ such that, for any $p \in U$ and $[a] \in \Omega_{p}$, there exists an (non-branched) $n$-end catenoid with the flux data $(p, a)$.

By the proposition, the inverse problem of the flux formula can be solved for almost all flux data if one succeeds to take such a point $c$. This will be done in the next section. To prove the proposition, we shall prepare several lemmas.

By the condition (4), at least one $(n-4)$-submatrix $S_{u_{0}}$ of $J_{u_{0}}$ is of rank $n-4$. Let $1 \leq j_{1}<j_{2}<\cdots<j_{n-4}<n$ be the indices of the columns of the submatrix $S_{u_{0}}$, and $\left\{m_{1}, m_{2}, m_{3}\right\}$ their complement, namely $\left\{m_{1}, m_{2}, m_{3}\right\}=\{1, \ldots, n-1\} \backslash\left\{j_{1}, \ldots, j_{n-4}\right\}$. By Remark 1.3, we may restrict the flux map into the following subspace of $\mathbf{P}^{n-1}$ containing the sampling point $c$ :

$$
\begin{aligned}
& \mathcal{V}^{n-3}:= \\
& \quad\left\{\left[q_{1}, \ldots, q_{n}\right] \in \mathbf{P}^{n-1} ; c_{m_{2}} q_{m_{1}}-c_{m_{1}} q_{m_{2}}=0, c_{m_{3}} q_{m_{1}}-c_{m_{1}} q_{m_{3}}=0\right\} .
\end{aligned}
$$


Now we define a homogeneous polynomial in $q_{1}, \ldots, q_{n}$ by

$$
H_{p}(q):=\Delta(q)^{2} \frac{\partial \operatorname{det} A_{p}}{\partial q_{n}}(q) \cdot \operatorname{det}\left(\Delta(q)^{l} S_{p}(q)\right) \cdot R_{p}(q) \cdot \prod_{j=1}^{n} f \ell_{p}^{j}(q) \cdot \prod_{k=1}^{n-1} q_{k}
$$

where $l$ is chosen sufficiently large so that $\operatorname{det}\left(\Delta(q)^{l} S_{p}(q)\right)$ is a homogeneous polynomial in $q_{1}, \ldots, q_{n}$, and $R_{p}$ is the resultant of the two polynomials $P(z)$ and $Q(z)$ of degree $n-1$ defined by (1.6) and (1.5). (It can be easily shown that $R_{p}$ is also a homogeneous polynomial with respect to $q$. ) Then by the conditions (1)-(7), $c \in \mathcal{V}^{n-3}$ satisfies $H_{u_{0}}(c) \neq 0$. We prove the following

Lemma 1.8. The subset

$$
U:=\left\{p \in \mathbf{C}^{n} ; \mathrm{Z}\left(\lambda_{p}\right) \cap \mathcal{V}^{n-3} \not \subset \mathrm{Z}\left(H_{p}\right)\right\}
$$

is open dense in $\mathbf{C}^{n}$, where $\lambda_{p}=\operatorname{det}\left(\Delta \cdot A_{p}\right)$ is the homogeneous polynomial defined in Lemma 1.6.

Proof. Obviously $U$ is an open subset of $\mathbf{C}^{n}$. Suppose that $U$ is not dense in $\mathbf{C}^{n}$. Then there exists an open subset $V$ such that

$$
\mathrm{Z}\left(\left.\lambda_{p}\right|_{\mathcal{V}^{n-3}}\right) \subset \mathrm{Z}\left(\left.H_{p}\right|_{\mathcal{V}^{n-3}}\right) \quad(p \in V) .
$$

Since $\mathcal{V}^{n-3} \cong \mathbf{P}^{n-3}$, by Lemma A.1 in Appendix A, (1.14) holds for any $p \in \mathbf{C}^{n}$ such that $\lambda_{p} \not \equiv 0$. But this contradicts the fact that $\lambda_{u_{0}}(c)=0$, $\lambda_{u_{0}} \not \equiv 0$ and $H_{u_{0}}(c) \neq 0$.

Roughly speaking, if $\mathcal{F} \ell_{p}$ has no singularities and is of maximal rank, then it is surjective and we can find a pair $\left(q, b_{p}(q)\right)$ satisfying (1.3) and (1.4). But unfortunately, $\mathcal{F} \ell_{p}$ has singularities on $\bigcap_{j=1}^{n} \mathrm{Z}\left(f \ell_{p}^{j}\right)$. For this reason, we define a new variety $\hat{\mathcal{V}}^{n-3}$ and a map $\widehat{\mathcal{F} \ell_{p}}: \hat{\mathcal{V}}^{n-3} \rightarrow \mathcal{W}_{p}^{n-4}$ instead of $\mathcal{V}^{n-3}$ 
and $\mathcal{F} \ell_{p}$ as follows. First we consider an algebraic set

$$
\begin{aligned}
& \mathcal{Y}^{n-3}=\left\{\left(\left[q_{1}, \ldots, q_{n}\right],\left[a^{1}, \ldots, a^{n}\right]\right) \in \mathbf{P}^{n-1} \times \mathbf{P}^{n-1} ;\right. \\
& c_{m_{2}} q_{m_{1}}-c_{m_{1}} q_{m_{2}}=0, c_{m_{3}} q_{m_{1}}-c_{m_{1}} q_{m_{3}}=0, \\
& a^{j} f \ell_{p}^{k}=a^{k} f \ell_{p}^{j} \quad(j, k=1, \ldots, n), \\
& \sum_{j=1}^{n} \frac{\left|p_{j}\right|^{2}-1}{\left|p_{j}\right|^{2}+1} a^{j}=0, \sum_{j=1}^{n} \frac{p_{j}}{\left|p_{j}\right|^{2}+1} a^{j}=0, \sum_{j=1}^{n} \frac{\overline{p_{j}}}{\left|p_{j}\right|^{2}+1} a^{j}=0 \\
&(j=1, \ldots, n)\},
\end{aligned}
$$

and define two canonical projections:

$$
\begin{aligned}
\pi: \mathcal{Y}^{n-3} \ni([q],[a]) & \mapsto[q] \in \mathcal{V}^{n-3}, \\
\pi^{\prime}: \mathcal{Y}^{n-3} \ni([q],[a]) & \mapsto[a] \in \mathcal{W}_{p}^{n-4} .
\end{aligned}
$$

These two projections are both well-defined on $\mathcal{Y}^{n-3}$. Let $\hat{\mathcal{V}}^{n-3}$ be the algebraic closure of the set

$$
\hat{\mathcal{V}}_{\text {reg }}^{n-3}:=\pi^{-1}\left(\mathcal{V}^{n-3} \backslash \bigcap_{j=1}^{n} \mathrm{Z}\left(f \ell_{p}^{j}\right)\right) .
$$

We denote the restriction of the first projection $\pi$ to $\hat{\mathcal{V}}^{n-3}$ also by $\pi$. We remark that $\left.\pi\right|_{\hat{\mathcal{V}}_{\text {reg }}^{n-3}}: \hat{\mathcal{V}}_{\text {reg }}^{n-3} \rightarrow \mathcal{V}^{n-3} \backslash \bigcap_{j=1}^{n} \mathrm{Z}\left(f \ell_{p}^{j}\right)$ is bijective. On the other hand, we denote the restriction of the second projection $\pi^{\prime}$ to $\hat{\mathcal{V}}^{n-3}$ by

$$
\widehat{\mathcal{F} \ell_{p}}: \hat{\mathcal{V}}^{n-3} \rightarrow \mathcal{W}_{p}^{n-4} \text {. }
$$

The map $\mathcal{F} \ell_{p} \circ \pi$ is well-defined on $\hat{\mathcal{V}}_{\text {reg }}^{n-3}$, and coincides with the map $\widehat{\mathcal{F} \ell_{p}}$.

Lemma 1.9. For each $p \in U$ satisfying $\operatorname{dim} \mathcal{W}_{p}^{n-4}=n-4$, there exists an irreducible component $\hat{X}^{n-4}$ of the algebraic set $\mathrm{Z}\left(\lambda_{p} \circ \pi\right) \cap \hat{\mathcal{V}}^{n-3}$ such that $H_{p} \circ \pi$ is not identically zero on $\hat{X}^{n-4}$. In addition, the restriction of the lifted flux map $\left.\widehat{\mathcal{F} \ell_{p}}\right|_{\hat{X}^{n-4}}: \hat{X}^{n-4} \rightarrow \mathcal{W}_{p}^{n-4}$ is surjective.

Proof. Suppose that $\mathrm{Z}\left(\lambda_{p} \circ \pi\right) \cap \hat{\mathcal{V}}^{n-3} \subset \mathrm{Z}\left(H_{p} \circ \pi\right)$. Since $H_{p}$ is identically zero on the singular set $\bigcap_{j=1}^{n} \mathrm{Z}\left(f \ell_{p}^{j}\right)$, it follows that

$$
\mathrm{Z}\left(\lambda_{p}\right) \cap \mathcal{V}^{n-3} \subset \mathrm{Z}\left(H_{p}\right) .
$$


But this contradicts Lemma 1.8. Hence there exists an irreducible component $\hat{X}^{n-4}$ of the algebraic set $\mathrm{Z}\left(\lambda_{p} \circ \pi\right) \cap \hat{\mathcal{V}}^{n-3}$ such that $H_{p} \circ \pi$ is not identically zero on $\hat{X}^{n-4}$. We set

$$
X^{n-4}:=\pi\left(\hat{X}^{n-4}\right) .
$$

Now we take a point $x_{0} \in X^{n-4}$ such that $H_{p}\left(x_{0}\right) \neq 0$. Consequently, we have $x_{0} \notin \bigcap_{j=1}^{n} \mathrm{Z}\left(f \ell_{p}^{j}\right)$ and so $\mathcal{F} \ell_{p}\left(x_{0}\right) \in \mathcal{W}_{p}^{n-4}$ is defined. We remark here that the $m_{1}$-th component of $x_{0}$ in the homogeneous coordinates is not equal to zero. Now we take a coordinate of $\mathbf{P}^{n-1}$ around $x_{0}$ defined by

$$
\begin{aligned}
\varphi: \mathbf{C}^{n-1} \ni x=\left(x_{1}, \ldots\right. & \left., x_{m_{1}-1}, x_{m_{1}+1}, \ldots, x_{n}\right) \\
& \mapsto q=\left[x_{1}, \ldots, x_{m_{1}-1}, 1, x_{m_{1}+1}, \ldots, x_{n}\right] \in \mathbf{P}^{n-1} .
\end{aligned}
$$

Since we chose $x_{0}$ so that $H_{p}\left(x_{0}\right) \neq 0$, it holds that the derivative $\frac{\partial \operatorname{det} A_{p}}{\partial q_{n}}$ does not vanish at $x_{0}$. So by the implicit function theorem, there exists a function $Q_{n}$ defined on a sufficiently small neighborhood of $x_{0}$ such that

$$
\begin{aligned}
& \lambda_{p}\left(x_{1}, \ldots, x_{m_{1}-1}, 1, x_{m_{1}+1}, \ldots, x_{n-1}, Q_{n}(x)\right) \\
&=\operatorname{det} A_{p}\left(x_{1}, \ldots, x_{m_{1}-1}, 1, x_{m_{1}+1}, \ldots, x_{n-1}, Q_{n}(x)\right)=0 .
\end{aligned}
$$

Since

$$
x_{m_{1}}=1, \quad x_{m_{2}}=\frac{c_{m_{2}}}{c_{m_{1}}}, \quad x_{m_{3}}=\frac{c_{m_{3}}}{c_{m_{1}}} \quad \text { on } \quad \mathcal{V}^{n-3},
$$

$\left(x_{j_{1}}, \ldots, x_{j_{n-4}}\right)$ is considered as a local coordinate system of the variety $X^{n-4}$ around the regular point $x_{0}$. Since

$$
\frac{\partial Q_{n}}{\partial x_{j_{l}}}=-\frac{\partial \operatorname{det} A_{p}}{\partial q_{j_{l}}} / \frac{\partial \operatorname{det} A_{p}}{\partial q_{n}} \quad(l=1, \ldots, n-4)
$$

holds, one can easily check that the condition $\operatorname{det} S_{p}\left(x_{0}\right) \neq 0$ implies that the matrix

$$
\left(\frac{\partial(\stackrel{\circ}{f} \circ \circ \varphi)}{\partial x_{j_{l}}}+\frac{\partial Q_{n}}{\partial x_{j_{l}}} \frac{\partial\left(\stackrel{\circ}{\left.f_{p}^{k} \circ \varphi\right)}\right.}{\partial x_{n}}\right)_{k=1, \ldots, n-1 ; l=1, \ldots, n-4}
$$

is of rank $n-4$ at $x_{0}$. Hence the Jacobi matrix of $\mathcal{F} \ell_{p}$ is of rank $n-4$ at $x_{0}$, and so is that of $\widehat{\mathcal{F} \ell_{p}}$ at $\pi^{-1}\left(x_{0}\right)$. Thus by the proper mapping theorem, $\widehat{\mathcal{F} \ell_{p}}\left(\hat{X}^{n-4}\right)$ is an analytic subset of dimension $n-4$ in the same dimensional complex projective space $\mathcal{W}_{p}^{n-4}$. Hence $\widehat{\mathcal{F} \ell_{p}}\left(\hat{X}^{n-4}\right)=\mathcal{W}_{p}^{n-4}$. 
Lemma 1.10. Let $\mathcal{W}_{\mathbf{R}}=\left\{[a] \in \mathcal{W}_{p}^{n-4} ; a_{j} \in \mathbf{R}\right\}$. Then

$$
\left\{\mathcal{W}_{p}^{n-4} \backslash \widehat{\mathcal{F} \ell_{p}}\left(\mathrm{Z}\left(H_{p} \circ \pi\right) \cap \hat{X}^{n-4}\right)\right\} \cap \mathcal{W}_{\mathbf{R}}
$$

is an open dense subset in $\mathcal{W}_{\mathbf{R}}$.

Proof. By the proper mapping theorem and the theorem of Chow, $\widehat{\mathcal{F} \ell_{p}}\left(\mathrm{Z}\left(H_{p} \circ\right.\right.$ $\pi)$ ) is an algebraic subset of $\mathcal{W}_{p}^{n-4}$. Thus it is a closed subset in $\mathcal{W}_{p}^{n-4}$ in the usual topology. Hence

$$
\left\{\mathcal{W}_{p}^{n-4} \backslash \widehat{\mathcal{F} \ell_{p}}\left(\mathrm{Z}\left(H_{p} \circ \pi\right) \cap \hat{X}^{n-4}\right)\right\} \cap \mathcal{W}_{\mathbf{R}}
$$

is an open subset in $\mathcal{W}_{\mathbf{R}}$. Suppose that it is not dense in $\mathcal{W}_{\mathbf{R}}$. We may assume that $\widehat{\mathcal{F} l_{p}}\left(\mathrm{Z}\left(H_{p} \circ \pi\right) \cap \hat{X}^{n-4}\right)$ is common zeros of some homogeneous polynomials $\bigcap_{j=1}^{r} \mathrm{Z}\left(h_{j}\right)$. Then there exists an open subset in $\mathcal{W}_{p}^{n-4}$ on which each $h_{j}$ is identically zero. Since $\mathcal{W}_{\mathbf{R}}$ is a totally real subset of the complex projective space $\mathcal{W}_{p}^{n-4}$, by Lemma A.2 in Appendix A we have

$$
h_{1}=\cdots=h_{r}=0 \quad \text { on } \quad \mathcal{W}_{p}^{n-4} .
$$

This implies that $\widehat{\mathcal{F} \ell_{p}}\left(\mathrm{Z}\left(H_{p} \circ \pi\right) \cap \hat{X}^{n-4}\right)=\mathcal{W}_{p}^{n-4}$. So it holds that

$$
\begin{aligned}
n-4 & =\operatorname{dim} \mathcal{W}_{p}^{n-4}=\operatorname{dim} \widehat{\mathcal{F} \ell_{p}}\left(\mathrm{Z}\left(H_{p} \circ \pi\right) \cap \hat{X}^{n-4}\right) \\
& \leq \operatorname{dim} \mathrm{Z}\left(H_{p} \circ \pi\right) \cap \hat{X}^{n-4} \leq \operatorname{dim} \hat{X}^{n-4}=n-4 .
\end{aligned}
$$

By the irreducibility of $\hat{X}^{n-4}$, we have $\mathrm{Z}\left(H_{p} \circ \pi\right) \cap \hat{X}^{n-4}=\hat{X}^{n-4}$. But this contradicts the fact that $H_{p}\left(x_{0}\right) \neq 0$.

Proof of Proposition 1.7. Let $p$ be a point in $U$ satisfying $\operatorname{dim} \mathcal{W}_{p}^{n-4}=n-4$. As we mentioned before, $\operatorname{dim} \mathcal{W}_{p}^{n-4}=n-4$ holds on an open dense subset of $\left\{p \in \mathbf{C}^{n}\right\}$. Then for any

$$
[a] \in\left(\mathcal{W}_{p}^{n-4} \backslash \widehat{\mathcal{F} \ell_{p}}\left(\mathrm{Z}\left(H_{p} \circ \pi\right) \cap \hat{X}^{n-4}\right)\right) \cap \mathcal{W}_{\mathbf{R}},
$$

there exists $x \in X^{n-4} \backslash \mathrm{Z}\left(H_{p}\right)$ such that $\mathcal{F} \ell_{p}(x)=[a]$ by Lemma 1.9 and Lemma 1.10. Since $f \ell_{p}^{j}(x) \neq 0$ and also the resultant $R_{p}(x)$ does not vanish, $\left(x, b_{p}(x)\right)$ induces an $n$-end catenoid with the flux data $(p, a)$ by Theorem 1.1. 
For the later application, the following modification of Proposition 1.7 is needed: Recall here that any elements of the matrices $A_{p}$ and $J_{p}$ are rational functions in $p_{1}, \ldots, p_{n}, \bar{p}_{1}, \ldots, \bar{p}_{n}$ and $q_{1}, \ldots, q_{n}$. Let $\check{A}_{p}$ and $\breve{J}_{p}$ be the matrices obtained by replacing $\bar{p}_{n}$ by $p_{n}$, namely

$$
\begin{aligned}
\check{A}_{p} & :=A_{p}\left(p_{1}, \ldots, p_{n}, \bar{p}_{1}, \ldots, \bar{p}_{n-1}, p_{n}, q_{1}, \ldots, q_{n}\right), \\
\check{J}_{p} & :=J_{p}\left(p_{1}, \ldots, p_{n}, \bar{p}_{1}, \ldots, \bar{p}_{n-1}, p_{n}, q_{1}, \ldots, q_{n}\right),
\end{aligned}
$$

and let $\check{b}_{p}^{j}$ (resp. $\check{f}_{p}^{j}, \check{\mathcal{W}}_{p}^{n-4}$ ) be the vector (resp. function, set) obtained by replacing $\bar{p}_{n}$ in $b_{p}^{j}$ (resp. $\left.f_{p}^{j}, \mathcal{W}_{p}^{n-4}\right)$ by $p_{n}$.

Proposition 1.11. Suppose that there exist $u_{0} \in \mathbf{C}^{n-1} \times \mathbf{R}$ and a point $c=\left[c_{1}, \ldots, c_{n}\right] \in \mathbf{P}^{n-1}$ satisfying the following conditions:

(1) $c_{1}, \ldots, c_{n}$ are all distinct;

(2) The rank of the matrix $\check{A}_{u_{0}}(c)$ is $n-1$;

(3) $\partial \operatorname{det} \check{A}_{u_{0}} / \partial q_{n}$ does not vanish at $q=c$;

(4) The rank of the matrix $\check{J}_{u_{0}}(c)$ is $n-4$;

(5) Two polynomials $P(z)$ and $Q(z)$ defined in (1.6) and (1.5) associated with the data $(q, p)=\left(c, u_{0}\right)$ and $\breve{b}=\check{b}_{u_{0}}(c)$ are mutually prime and one of them has degree $n-1$;

(6) $\check{f}_{u_{0}}^{j}(c) \neq 0(j=1, \ldots, n)$;

(7) $c_{j} \neq 0(j=1, \ldots, n-1)$.

Then there exists an open dense subset $U \subset \mathbf{C}^{n-1} \times \mathbf{R}$ and an open dense subset $\Omega_{p}$ of the totally real set $\mathcal{W}_{\mathbf{R}}=\left\{[a] \in \check{\mathcal{W}}_{p}^{n-4} ; a_{j} \in \mathbf{R}\right\}$ such that, for $p=\left(p_{1}, \ldots, p_{n}\right) \in U$ and $[a] \in \Omega_{p}$, there exists an (non-branched) $n$-end catenoid with the flux data $(p, a)$.

Proof. The proof of Proposition 1.7 works even if we replace $\bar{p}_{n}$ by $p_{n}$. When $p_{n}$ is real, $\check{A}_{p}, \check{J}_{p}, \check{\mathcal{F}} \ell_{p}$ and $\check{\mathcal{W}}_{p}^{n-4}$ coincide with $A_{p}, J_{p}, \mathcal{F} \ell_{p}$ and $\mathcal{W}_{p}^{n-4}$ respectively. In fact, by the same proof as Lemma 1.7, we can prove that $U:=\left\{p \in \mathbf{C}^{n-1} \times \mathbf{R} ; \mathrm{Z}\left(\lambda_{p}\right) \cap \mathcal{V}^{n-3} \not \subset \mathrm{Z}\left(H_{p}\right)\right\}$ is open dense in $\mathbf{C}^{n-1} \times \mathbf{R}$, because we only need the real analyticity with respect to the parameter $p$ for applying Lemma A.1. 
Remark 1.12. To solve the inverse problem of the flux formula, we may assume that $p_{n} \in \mathbf{R}$ since by a suitable rotation in $\left\{(x, y, z) \in \mathbf{R}^{3}\right\}$, we can choose that $v_{n}$ is in the $x z$-plane.

\section{Finding a regular point of the flux map.}

In the previous section, we reduced our inverse problem to finding a regular point of the flux map. However, the following difficulties arise in this process.

- As seen in [Kat] and [KUY1], $n$-end catenoids with many symmetries are easy to construct. But unfortunately, they are not expected to be a regular point of the flux map because of their symmetries.

- If we take a less symmetric $n$-end catenoid, the computation of the rank of the flux map is very complicated and hard to calculate even by computer.

To avoid these difficulties, we first take an $n$-end catenoid with many symmetries, and next consider a perturbation of it which attains the desired properties.

Set $m:=n-1$. First we consider a 1-parameter family of $(m+1)$-end catenoids given in [Kat];

$$
\left\{\begin{array}{lll}
p_{j}:=r \zeta^{j-1} & (j=1, \ldots, m), & p_{m+1}:=0 \\
a^{j}:=\frac{m-1}{2} r\left(r^{2}+1\right) & (j=1, \ldots, m), & a^{m+1}:=\frac{m(m-1)}{2} r\left(r^{2}-1\right), \\
q_{j}:=\zeta^{j-1} & (j=1, \ldots, m), & q_{m+1}:=0 \\
b^{j}:=1, & (j=1, \ldots, m), & b^{m+1}:=\frac{m-1}{2}\left(r^{2}-1\right),
\end{array}\right.
$$

where $r>0, r \neq 1$ and $\zeta:=\exp (2 \pi \sqrt{-1} / m)$. In fact, they are $(m+1)$-end catenoids without branch points by Remark 1.2, and are invariant under the action of the cyclic group $Z_{m}$. Unfortunately, as we shall see below, $J_{p}(q)=\breve{J}_{p}(q)=0$ holds for any of these examples, namely they all are singular points of the flux maps. However, we will show that there exists a regular point near them. 
Note here that the matrix $A_{p}(q)$ (defined in (1.10)) for the example above is given by

$$
A_{p}(q)=\left(\begin{array}{ccccc}
0 & \frac{1+r^{2} \zeta^{1}}{q_{1}-q_{2}} & \ldots & \frac{1+r^{2} \zeta^{m-1}}{q_{1}-q_{m}} & \frac{1}{q_{1}-q_{m+1}} \\
\frac{1+r^{2} \zeta^{-1}}{q_{2}-q_{1}} & 0 & \ldots & \frac{1+r^{2} \zeta^{m-2}}{q_{2}-q_{m}} & \frac{1}{q_{2}-q_{m+1}} \\
\vdots & \vdots & \ddots & \vdots & \vdots \\
\frac{1+r^{2} \zeta^{-(m-1)}}{q_{m}-q_{1}} & \frac{1+r^{2} \zeta^{-(m-2)}}{q_{m}-q_{2}} & \ldots & 0 & \frac{1}{q_{m}-q_{m+1}} \\
\frac{1}{q_{m+1}-q_{1}} & \frac{1}{q_{m+1}-q_{2}} & \ldots & \frac{1}{q_{m+1}-q_{m}} & 0
\end{array}\right) .
$$

Now, we consider a 1-parameter family of matrices

$$
A(q, \mu):=\left(\begin{array}{ccccc}
0 & \frac{1+\mu \zeta^{1}}{q_{1}-q_{2}} & \ldots & \frac{1+\mu \zeta^{m-1}}{q_{1}-q_{m}} & \frac{1}{q_{1}-q_{m+1}} \\
\frac{1+\mu \zeta^{-1}}{q_{2}-q_{1}} & 0 & \ldots & \frac{1+\mu \zeta^{m-2}}{q_{2}-q_{m}} & \frac{1}{q_{2}-q_{m+1}} \\
\vdots & \vdots & \ddots & \vdots & \vdots \\
\frac{1+\mu \zeta^{-(m-1)}}{q_{m}-q_{1}} & \frac{1+\mu \zeta^{-(m-2)}}{q_{m}-q_{2}} & \cdots & 0 & \frac{1}{q_{m}-q_{m+1}} \\
\frac{1}{q_{m+1}-q_{1}} & \frac{1}{q_{m+1}-q_{2}} & \cdots & \frac{1}{q_{m+1}-q_{m}} & 0
\end{array}\right) .
$$

By comparing (2.2) with (2.3), we have $A\left(q, r^{2}\right)=A_{p}(q)$ for $p$ as in (2.1). When we evaluate it at $q=q^{0}:=\left(1, \zeta^{1}, \ldots, \zeta^{m-1}, 0\right)$, we have

(2.4) $A\left(q^{0}, \mu\right)=\left(\begin{array}{ccccc}0 & \frac{1+\mu \zeta^{1}}{1-\zeta^{1}} & \ldots & \frac{1+\mu \zeta^{m-1}}{1-\zeta^{m-1}} & 1 \\ \frac{1+\mu \zeta^{-1}}{\zeta^{1}-1} & 0 & \ldots & \frac{1+\mu \zeta^{m-2}}{\zeta^{1}-\zeta^{m-1}} & \zeta^{-1} \\ \vdots & \vdots & \ddots & \vdots & \vdots \\ \frac{1+\mu \zeta^{-(m-1)}}{\zeta^{m-1}-1} & \frac{1+\mu \zeta^{-(m-2)}}{\zeta^{m-1}-\zeta^{1}} & \ldots & 0 & \zeta^{-(m-1)} \\ -1 & -\zeta^{-1} & \ldots & -\zeta^{-(m-1)} & 0\end{array}\right)$.

We remark that the matrix $A\left(q^{0}, \mu\right)$ has the simplest form when $\mu=-1$. The following lemma holds.

Lemma 2.1. The $(m+1)$-matrix $A\left(q^{0}, \mu\right)$ is of rank $m$ except for finite values of $\mu \in \mathbf{R}$. Moreover $A\left(q^{0}, \mu\right)$ has a 0 -eigenvector given by

$$
t\left(1, \ldots, 1, \frac{m-1}{2}(\mu-1)\right) .
$$


Proof. The second assertion is easily checked. Hence the rank of the matrix $A\left(q^{0},-1\right)$ is at most $m$. Moreover, it is easy to see that the rank of the matrix $A\left(q^{0},-1\right)$ is $m$. Since each component of $A\left(q^{0}, \mu\right)$ is a polynomial in $\mu$, the first assertion is obtained.

Remark 2.2. Similarly, a 0-eigenvector of ${ }^{t} A\left(q^{0}, \mu\right)$ is given by

$$
{ }^{t}\left(1, \ldots, 1, \frac{1}{2}\{2 \mu-(m-1)(\mu+1)\}\right) .
$$

Proposition 2.3. The following identity holds.

$$
\frac{\partial \operatorname{det} A}{\partial q_{j}}\left(q^{0}, \mu\right)=0 \quad(j=1, \ldots, m+1) .
$$

Proof. We denote the cofactor matrix of $A(q, \mu)$ by $B(q, \mu)$. By Lemma 2.1 and Remark 2.2 , it can be easily checked that $B\left(q^{0}, \mu\right)$ is written in the form $B\left(q^{0}, \mu\right)=f(\mu) S(\mu)$, where $f(\mu)$ is a polynomial in $\mu$ satisfying $f(-1)=1$,

$$
S(\mu):=\left(\begin{array}{cccc}
1 & \cdots & 1 & \psi(\mu) \\
\vdots & \ddots & \vdots & \vdots \\
1 & \cdots & 1 & \psi(\mu) \\
\varphi(\mu) & \cdots & \varphi(\mu) & \varphi(\mu) \cdot \psi(\mu)
\end{array}\right)
$$

and $\varphi(\mu)$ and $\psi(\mu)$ are explicitly given by

$$
\varphi(\mu):=\frac{m-1}{2}(\mu-1), \quad \psi(\mu):=\frac{1}{2}\{2 \mu-(m-1)(\mu+1)\} .
$$

Note here that

$$
\frac{\partial \operatorname{det} A}{\partial q_{j}}(q, \mu)=\operatorname{Tr}\left(\frac{\partial A}{\partial q_{j}}(q, \mu) \cdot B(q, \mu)\right)
$$

always holds for any $j$. Denote the $(k, l)$-component of the matrix $A(q, \mu)$ by $\alpha_{k l}(q, \mu)$. Then we have

$$
\frac{\partial \alpha_{k l}}{\partial q_{j}}\left(q^{0}, \mu\right)= \begin{cases}-\frac{1+\mu \zeta^{l-j}}{\left(\zeta^{j-1}-\zeta^{l-1}\right)^{2}} & (k=j ; l=1, \ldots, m ; l \neq j) \\ -\zeta^{-2(j-1)} & (k=j ; l=m+1) \\ \frac{1+\mu \zeta^{j-k}}{\left(\zeta^{k-1}-\zeta^{j-1}\right)^{2}} & (k=1, \ldots, m ; k \neq j ; l=j) \\ \zeta^{-2(j-1)} & (k=m+1 ; l=j) \\ 0 & \text { elsewhere }\end{cases}
$$


for $j=1, \ldots, m$, and

$$
\frac{\partial \alpha_{k l}}{\partial q_{m+1}}\left(q^{0}, \mu\right)= \begin{cases}\zeta^{-2(k-1)} & (k=1, \ldots, m ; l=m+1) \\ -\zeta^{-2(l-1)} & (k=m+1 ; l=1, \ldots, m) \\ 0 & \text { elsewhere }\end{cases}
$$

for $j=m+1$.

For $j=1, \ldots, m$, by using the formula above, we have

$$
\left.\begin{array}{rl}
\frac{\partial \operatorname{det} A}{\partial q_{j}}\left(q^{0}, \mu\right)=\operatorname{Tr}\left(\frac{\partial A}{\partial q_{j}}\left(q^{0}, \mu\right) \cdot B\left(q^{0}, \mu\right)\right) & \\
= & \sum_{\substack{k=1 \\
k \neq j}}^{m} f(\mu) \frac{\partial \alpha_{k j}}{\partial q_{j}}\left(q^{0}, \mu\right)+\sum_{\substack{l \neq 1 \\
l \neq j}}^{m} f(\mu) \frac{\partial \alpha_{j l}}{\partial q_{j}}\left(q^{0}, \mu\right) \\
& +\frac{\partial \alpha_{j m+1}}{\partial q_{j}}\left(q^{0}, \mu\right) f(\mu) \varphi(\mu)+\frac{\partial \alpha_{m+1 j}}{\partial q_{j}}\left(q^{0}, \mu\right) f(\mu) \psi(\mu) \\
= & f(\mu)\left\{\sum_{\substack{k=1 \\
k \neq j}}^{m} \frac{1+\mu \zeta^{j-k}}{\left.\zeta^{k-1}-\zeta^{j-1}\right)^{2}}-\sum_{\substack{l \neq 1 \\
l \neq j}}^{m} \frac{1+\mu \zeta^{l-j}}{\left(\zeta^{j-1}-\zeta^{l-1}\right)^{2}}\right. \\
= & f(\mu) \zeta^{-2(j-1)}\left\{\sum_{k=1}^{m-1} \frac{1+\mu \zeta^{-k}}{\left(1-\zeta^{k}\right)^{2}}-\sum_{k=1}^{m-1} \frac{1+\mu \zeta^{k}}{\left(1-\zeta^{k}\right)^{2}}-(m-2) \mu\right\} \\
= & \mu f(\mu) \zeta^{-2(j-1)}\left\{\sum_{k=1}^{m-1} \frac{1+\zeta^{k}}{\zeta^{k}\left(1-\zeta^{k}\right)}-(m-2)\right\} \\
= & \mu f(\mu) \zeta^{-2(j-1)}\left\{\sum_{k=1}^{m-1} \frac{1}{\zeta^{k}}+\sum_{k=1}^{m-1} \frac{2}{1-\zeta^{k}}-(m-2)\right\} \\
= & \mu f(\mu) \zeta^{-2(j-1)}\{-1+(m-1)-(m-2)\}=0 .
\end{array}\right\}
$$


On the other hand, for $j=m+1$, we have

$$
\begin{aligned}
\frac{\partial \operatorname{det} A}{\partial q_{m+1}}\left(q^{0}, \mu\right) & =\operatorname{Tr}\left(\frac{\partial A}{\partial q_{m+1}}\left(q^{0}, \mu\right) \cdot B\left(q^{0}, \mu\right)\right) \\
& =\sum_{k=1}^{m} \zeta^{-2(k-1)} f(\mu) \varphi(\mu)-\sum_{l=1}^{m} \zeta^{-2(l-1)} f(\mu) \psi(\mu) \\
& =f(\mu)(\varphi(\mu)-\psi(\mu)) \sum_{k=1}^{m} \zeta^{-2 k}=0
\end{aligned}
$$

This completes the proof.

By Lemma 2.1 and Proposition 2.3, it follows that $J_{r q^{0}}\left(q^{0}\right)=0(r \in \mathbf{R})$. Therefore, we try to perturb a sampling point. To do this, we consider an $m$-matrix $\Gamma_{m+1}(\mu)$ by

$$
\begin{aligned}
\Gamma_{m+1}(\mu):= & \left(\frac{\partial^{2} \operatorname{det} A}{\partial q_{1} \partial q_{m+1}}\left(q^{0}, \mu\right) \cdot \frac{\partial\left(f^{k} / f^{m+1}\right)}{\partial q_{j}}\left(q^{0}, \mu\right)\right. \\
- & \left.\frac{\partial^{2} \operatorname{det} A}{\partial q_{1} \partial q_{j}}\left(q^{0}, \mu\right) \cdot \frac{\partial\left(f^{k} / f^{m+1}\right)}{\partial q_{m+1}}\left(q^{0}, \mu\right)\right)_{j, k=1, \ldots, m}
\end{aligned}
$$

where we denote the $(j, k)$-component of the cofactor matrix $B(q, \mu)$ by $\beta_{j k}(q, \mu)$, and set

$$
\begin{aligned}
& f^{k}(q, \mu):=\beta_{k m+1}(q, \mu) \\
& \times\left(\sum_{\substack{j \neq 1 \\
j \neq k}}^{m} \beta_{j m+1}(q, \mu) \frac{\zeta^{k-1}-\zeta^{j-1}}{q_{k}-q_{j}}+\beta_{m+1 m+1}(q, \mu) \frac{\zeta^{k-1}}{q_{k}-q_{m+1}}\right) \\
& f^{m+1}(q, \mu)\left.\left.:=\beta_{m+1}\right), \ldots, m\right), \\
&(k=1, \ldots, \mu) \sum_{j=1}^{m} \beta_{j m+1}(q, \mu) \frac{-\zeta^{j-1}}{q_{m+1}-q_{j}} .
\end{aligned}
$$

(Compare with the definition of the matrix $J_{p}(q)$ and $f_{p}^{k}(q)$.) We prove the following 
Theorem 2.4. Suppose that there exists a positive number $\mu$ such that the matrix $\Gamma_{m+1}(\mu)(n=m+1 \geq 5)$ is of rank $m-3(=n-4)$ and

$$
\frac{\partial^{2} \operatorname{det} A}{\partial q_{1} \partial q_{m+1}}\left(q^{0}, \mu\right) \neq 0 .
$$

Then, for each of almost all flux data, there exists an n-end catenoid with the flux data.

Till now, we fix the parameter $p_{m+1}$ at

$$
p_{m+1}=0 .
$$

Let us now move $p_{m+1}$ as a complex parameter.

Lemma 2.5. Let $\mu \neq 1$ be a positive real number such that $f(\mu) \neq 0$, where $f(\mu)$ is a polynomial given by (2.5). Then

$$
\frac{\partial \operatorname{det} \check{A}_{p}(q)}{\partial p_{m+1}} \neq 0
$$

at the point $q=q^{0}=\left(1, \zeta^{1}, \ldots, \zeta^{m-1}, 0\right)$ for $p=\sqrt{\mu} q^{0}$, where $\check{A}_{p}(q)$ is defined in (1.16).

Proof. We denote the cofactor matrix of $\check{A}_{p}(q)$ by $\check{B}_{p}(q)$. Since $\check{A}_{\sqrt{\mu} q^{0}}(q)=$ $A_{\sqrt{\mu} q^{0}}(q)$ for any $\mu>0$, it holds that $\check{B}_{\sqrt{\mu} q^{0}}(q)=B_{\sqrt{\mu} q^{0}}(q)$ and in particular, we have $\check{B}_{\sqrt{\mu} q^{0}}\left(q^{0}\right)=B_{\sqrt{\mu} q^{0}}\left(q^{0}\right)=B\left(q^{0}, \mu\right)$. Then we have

$$
\left.\frac{\partial \operatorname{det} \check{A}_{p}\left(q^{0}\right)}{\partial p_{m+1}}\right|_{p=\sqrt{\mu} q^{0}}=\operatorname{Tr}\left(\left.\frac{\partial \check{A}_{p}\left(q^{0}\right)}{\partial p_{m+1}}\right|_{p=\sqrt{\mu} q^{0}} \cdot B_{\sqrt{\mu} q^{0}}\left(q^{0}\right)\right) .
$$

Since

$$
\text { the } \begin{aligned}
&(j, k) \text {-component of }\left.\frac{\partial \check{A}_{p}\left(q^{0}\right)}{\partial p_{m+1}}\right|_{p=\sqrt{\mu} q^{0}} \\
&= \begin{cases}\zeta^{-2(j-1)} & (j=1, \ldots, m ; k=m+1) \\
-1 & (j=m+1 ; k=1, \ldots, m) \\
0 & \text { elsewhere }\end{cases}
\end{aligned}
$$


by $(2.5)$, we have

$$
\begin{aligned}
\operatorname{Tr}\left(\left.\frac{\partial \check{A}_{p}\left(q^{0}\right)}{\partial p_{m+1}}\right|_{p=\sqrt{\mu} q^{0}}\right. & \left.\cdot B\left(q^{0}, \mu\right)\right) \\
& =f(\mu)\left\{\varphi(\mu) \sum_{k=1}^{m} \zeta^{-2(k-1)}-(m-1) \psi(\mu)\right\} \\
& =-(m-1) f(\mu) \psi(\mu)=\frac{(m-1)^{2}}{2}(\mu-1) f(\mu) \neq 0
\end{aligned}
$$

Now the assertion is clear.

Proof of Theorem 2.4. Since $f(\mu)$ is a polynomial in $\mu$ and $f(\mu) \not \equiv 0$, by our assumptions and Lemmas 2.1 and 2.5, we can choose a positive number $\mu$ such that $f(\mu) \neq 0, \operatorname{rank} \check{A}_{\sqrt{\mu} q^{0}}\left(q^{0}\right)=m, \operatorname{rank} \Gamma_{m+1}(\mu)=m-3$,

$$
\frac{\partial^{2} \operatorname{det} \check{A}_{\sqrt{\mu} q^{0}}}{\partial q_{1} \partial q_{m+1}}\left(q^{0}\right) \neq 0 \quad \text { and }\left.\quad \frac{\partial \operatorname{det} \check{A}_{p}\left(q^{0}\right)}{\partial p_{m+1}}\right|_{p=\sqrt{\mu} q^{0}} \neq 0
$$

Throughout this proof, we fix the parameters except for $q_{1}$ and $p_{m+1}$ to the same values as $q=q^{0}$ and $p=\sqrt{\mu} q^{0}$ :

$$
\begin{array}{rlrl}
p_{j} & =\sqrt{\mu} \zeta^{j-1} & & (j=1, \ldots, m), \\
q_{j}=\zeta^{j-1} & & (j=2, \ldots, m),
\end{array}
$$

Regard $\operatorname{det} \check{A}_{p}(q)$ as a function with respect to only $q_{1}$ and $p_{m+1}$, and apply the implicit function theorem to the point $\left(q_{1}, p_{m+1}\right)=(1,0)$. Then there exist an open neighborhood $U \subset \mathbf{C}$ of $1 \in \mathbf{C}$ and a complex analytic function $p_{m+1}=p_{m+1}\left(q_{1}\right): U \rightarrow \mathbf{C}$ such that $p_{m+1}(1)=0$ and

$$
\left.\operatorname{det} \check{A}_{p}\right|_{p_{m+1}=p_{m+1}\left(q_{1}\right)}=0 \quad\left(q_{1} \in U\right) .
$$

Since $\operatorname{rank} \check{A}_{\sqrt{\mu} q^{0}}\left(q^{0}\right)=m,\left.\operatorname{rank} \check{A}_{p}\right|_{p_{m+1}=p_{m+1}\left(q_{1}\right)}=m$ holds also for $q_{1}$ near 1.

Since $\hat{A}=A$ at $p=\sqrt{\mu} q^{0}$, by Lemma 2.3 , we have

$$
\frac{\partial \operatorname{det} \check{A}_{\sqrt{\mu} q^{0}}}{\partial q_{j}}\left(q^{0}\right)=0 \quad(j=1, \ldots, m+1) .
$$


On the other hand, the assumption (2.7) yields

$$
\left.\frac{\partial \operatorname{det} \check{A}_{p}}{\partial q_{m+1}}\right|_{p_{m+1}=p_{m+1}\left(q_{1}\right)} \neq 0
$$

for any $q_{1} \neq 1$ enough close to 1 . Therefore we have

$$
\begin{aligned}
& \lim _{q_{1} \rightarrow 1}\left(\left.\left\{\frac{\partial\left(\check{f}_{p}^{k} / \check{f}_{p}^{m+1}\right)}{\partial q_{j}}-\frac{\frac{\partial \operatorname{det} \check{A}_{p}}{\partial q_{j}}}{\frac{\partial \operatorname{det} \check{A}_{p}}{\partial q_{m+1}}} \cdot \frac{\partial\left(\check{f}_{p}^{k} / \check{f}_{p}^{m+1}\right)}{\partial q_{m+1}}\right\}\right|_{p_{m+1}=p_{m+1}\left(q_{1}\right)}\right)_{j, k=1, \ldots, m} \\
& =\left(\left.\left\{\frac{\partial\left(\check{f}_{p}^{k} / \check{f}_{p}^{m+1}\right)}{\partial q_{j}}-\frac{\frac{\partial^{2} \operatorname{det} \check{A}_{p}}{\partial q_{1} \partial q_{j}}}{\frac{\partial^{2} \operatorname{det} \check{A}_{p}}{\partial q_{1} \partial q_{m+1}}} \cdot \frac{\partial\left(\check{f}_{p}^{k} / \check{f}_{p}^{m+1}\right)}{\partial q_{m+1}}\right\}\right|_{p=\sqrt{\mu} q^{0} ; q=q^{0}}\right)_{j, k=1, \ldots, m} \\
& =\left(\frac{\partial^{2} \operatorname{det} \check{A} \sqrt{\mu} q^{0}}{\partial q_{1} \partial q_{m+1}}\left(q^{0}\right)\right)^{-1} \Gamma_{m+1}(\mu),
\end{aligned}
$$

and hence

$$
\begin{aligned}
& \left.\operatorname{rank} \check{J}_{p}\right|_{p_{m+1}=p_{m+1}\left(q_{1}\right)} \\
& =\operatorname{rank}\left(\left.\left\{\frac{\partial\left(\check{f}_{p}^{k} / \check{f}_{p}^{m+1}\right)}{\partial q_{j}}-\frac{\frac{\partial \operatorname{det} \check{A}_{p}}{\partial q_{j}}}{\frac{\partial \operatorname{det} \check{A}_{p}}{\partial q_{m+1}}} \cdot \frac{\partial\left(\check{f}_{p}^{k} / \check{f}_{p}^{m+1}\right)}{\partial q_{m+1}}\right\}\right|_{p_{m+1}=p_{m+1}\left(q_{1}\right)}\right)_{j, k=1, \ldots, m} \\
& =m-3=n-4
\end{aligned}
$$

for any $q_{1}$ as above.

Since the initial sampling point $q=q^{0}, p=\sqrt{\mu} q^{0}$ is chosen from the data which realizes a non-branched $n$-end catenoid $(n=m+1), \Delta\left(q^{0}\right) \neq 0$ and $q_{j}^{0} \neq 0(j=1, \ldots, m)$, the other conditions in Proposition 1.11 are also satisfied for $q_{1}$ near 1 such that $p_{m+1} \in \mathbf{R}$. Now, by Remark 1.12, we have proved the theorem.

Thus we will get our main theorem in Introduction, if the matrix $\Gamma_{m+1}(\mu)$ is of rank $m-3(=n-4)$ and (2.7) holds for some $\mu>0$, which will be shown in the next section.

\section{Computation of $\Gamma_{m+1}(\mu)$.}

In this section, we compute the matrix $\Gamma_{m+1}(\mu)$ defined in the previous section, and show that it is of rank $m-3$ for almost all $\mu \in(0,1) \cup(1,+\infty)$. 
Computation of $\left(\partial f^{k} / \partial q_{j}\right)\left(q^{0}, \mu\right)$. As before, we write

$$
A(q, \mu)=:\left(\alpha_{k l}\right)_{k, l=1, \ldots, m+1}
$$

and

$$
B(q, \mu)=:\left(\beta_{k l}\right)_{k, l=1, \ldots, m+1} .
$$

By (2.6), (2.5) and straightforward calculations, we have, for any $k=$ $1, \ldots, m$,

$$
\frac{\partial f^{k}}{\partial q_{j}}=f \psi\left[(m-1+\varphi) \frac{\partial \beta_{k m+1}}{\partial q_{j}}+\sum_{\substack{l=1 \\ l \neq k}}^{m+1} \frac{\partial \beta_{l m+1}}{\partial q_{j}}+f \psi \zeta^{1-j} \eta_{1}\right]
$$

at $\left(q^{0}, \mu\right)$, where

$$
\eta_{1}(\mu):= \begin{cases}-\frac{m-1}{2}-\varphi(\mu) & (j=k) \\ \frac{1}{\zeta^{k-j}-1} & (j=1, \ldots, m ; j \neq k) \\ \zeta^{j-k} \varphi(\mu) & (j=m+1),\end{cases}
$$

and for $k=m+1$,

(3.2) $\frac{\partial f^{m+1}}{\partial q_{j}}$

$$
=f \psi\left[m \frac{\partial \beta_{m+1 m+1}}{\partial q_{j}}+\varphi \sum_{l=1}^{m} \frac{\partial \beta_{l m+1}}{\partial q_{j}}-\left\{\begin{array}{ll}
f \varphi \psi \zeta^{1-j} & (j=1, \ldots, m) \\
0 & (j=m+1)
\end{array}\right] .\right.
$$

Hence we have only to compute $f(\mu)$ and $\partial \beta_{k m+1} / \partial q_{j}\left(q^{0}, \mu\right)$. Denote the first $m \times m$-submatrix of $A\left(q^{0}, \mu\right)$ by $A^{0}(\mu)$. Clearly

$$
f \varphi \psi=\beta_{m+1 m+1}=\operatorname{det} A^{0} .
$$

Take the diagonal matrix

$$
C_{1}:=\operatorname{diag}\left[1, \zeta^{1}, \ldots, \zeta^{m-1}\right] .
$$

Since $C_{1} A^{0}$ is a cyclic matrix whose $(j, k)$-component is equal to $(1+$ $\left.\mu \zeta^{k-j}\right) /\left(1-\zeta^{k-j}\right)$, and whose diagonal components vanish, it can be diagonalized as

$$
C_{2}{ }^{-1} C_{1} A^{0} C_{2}=\operatorname{diag}\left[\psi_{1}, \ldots, \psi_{m}\right]
$$


where

$$
C_{2}:=\left(\begin{array}{cccc}
1 & 1 & \cdots & 1 \\
\zeta^{1} & \zeta^{2} & \cdots & 1 \\
\vdots & \vdots & \ddots & \vdots \\
\zeta^{m-1} & \zeta^{2(m-1)} & \cdots & 1
\end{array}\right)
$$

and the eigenvalues $\psi_{1}, \ldots, \psi_{m}$ of $C_{1} A^{0}$ are given by

$$
\begin{aligned}
\psi_{l}(\mu) & =\sum_{k=2}^{m} \frac{1+\mu \zeta^{k-1}}{1-\zeta^{k-1}}\left(\zeta^{l}\right)^{k-1} \\
& = \begin{cases}\left(l-\frac{m-1}{2}\right) \mu+\left(l-\frac{m+1}{2}\right) & (l=1, \ldots, m-1) \\
-\frac{m-1}{2} \mu+\frac{m-1}{2} & (l=m) .\end{cases}
\end{aligned}
$$

Now we have

$$
f \varphi \psi=(-1)^{m-1} \prod_{l=1}^{m} \psi_{l}
$$

Note here that $\psi_{1}=\psi$ and $\psi_{m}=-\varphi$ and that $\psi_{l}(\mu) \neq 0$ holds for any $\mu \in(0,1) \cup(1,+\infty)(l=1, \ldots, m)$.

To compute the derivatives $\partial B / \partial q_{j}\left(q^{0}, \mu\right)$ of the cofactor matrix $B(q, \mu)$, we apply the formula (B.2) in Appendix B by putting $X:=E_{m+1}$, where $E_{m+1}$ is the $(m+1)$-matrix given by

$$
E_{m+1}:=\left(\begin{array}{cccc}
0 & \cdots & 0 & 0 \\
\vdots & \ddots & \vdots & \vdots \\
0 & \cdots & 0 & 0 \\
0 & \cdots & 0 & 1
\end{array}\right) .
$$

For $A_{t}(q, \mu)=A(q, \mu)+t E_{m+1}$, we have already shown that

$$
\operatorname{det} A\left(q^{0}, \mu\right)=\frac{\partial \operatorname{det} A}{\partial q_{j}}\left(q^{0}, \mu\right)=0
$$

in Lemma 2.1 and Proposition 2.3. Moreover we have

$$
\operatorname{Tr}\left(E_{m+1} \cdot B\left(q^{0}, \mu\right)\right)=f(\mu) \varphi(\mu) \psi(\mu) \neq 0 .
$$


Thus we may apply (B.2), and get the following identity

$$
\begin{aligned}
\frac{\partial B}{\partial q_{j}}=\frac{1}{f \varphi \psi}\left\{\operatorname{Tr}\left(\left.\frac{\partial A}{\partial q_{j}} \cdot \frac{\partial Y_{t}}{\partial t}\right|_{t=0}\right) \cdot B\right. & \\
& \left.\quad-\left.\frac{\partial Y_{t}}{\partial t}\right|_{t=0} \cdot \frac{\partial A}{\partial q_{j}} \cdot B-\left.B \cdot \frac{\partial A}{\partial q_{j}} \cdot \frac{\partial Y_{t}}{\partial t}\right|_{t=0}\right\}
\end{aligned}
$$

at $\left(q^{0}, \mu\right)$, where $Y_{t}(\mu)$ is the cofactor matrix of $A\left(q^{0}, \mu\right)+t E_{m+1}$. The first $m \times m$-components of $\partial Y_{t}(\mu) /\left.\partial t\right|_{t=0}$ is given as the cofactor matrix of the first $m \times m$-components of $A\left(q^{0}, \mu\right)$, that is

$$
\begin{aligned}
\operatorname{det} A^{0} \cdot\left(A^{0}\right)^{-1} & =f \varphi \psi \cdot C_{2} \operatorname{diag}\left[\psi_{1}^{-1}, \ldots, \psi_{m}{ }^{-1}\right] C_{2}{ }^{-1} C_{1} \\
& =\frac{f \varphi \psi}{m} Y^{0}
\end{aligned}
$$

here, we put

$$
Y^{0}:=\left(\zeta^{k-1} \sum_{l=1}^{m} \zeta^{(j-k) l} \psi_{l}^{-1}\right)_{j, k=1, \ldots, m}
$$

The other components of $\partial Y_{t}(\mu) /\left.\partial t\right|_{t=0}$ vanish. Namely

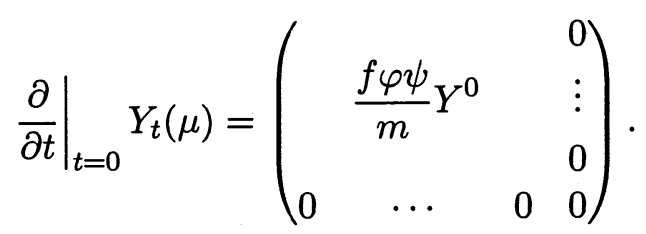

Therefore we have

$$
\left(\frac{\partial \beta_{k m+1}}{\partial q_{j}}\right)_{k=1, \ldots, m+1}=\frac{f \psi}{m}\left\{\operatorname{Tr}\left(\frac{\partial A}{\partial q_{j}} \cdot Y^{0}\right) \cdot I-Y^{0} \cdot \frac{\partial A}{\partial q_{j}}\right\}\left(\begin{array}{c}
1 \\
\vdots \\
1 \\
\varphi
\end{array}\right)
$$

at $\left(q^{0}, \mu\right)$. Recall here the values of $\left(\partial \alpha_{k l} / \partial q_{j}\right)\left(q^{0}, \mu\right)$ computed in the proof of Proposition 2.3. Now, by direct computation, we have

$$
\begin{aligned}
\frac{\partial \beta_{k m+1}}{\partial q_{j}}\left(q^{0}, \mu\right) & =-f(\mu) \psi(\mu) \zeta^{1-j} \\
& \times \begin{cases}\left(1-\frac{1}{2 m} \eta_{2}(\mu)\right) & (k, j=1, \ldots, m) \\
\varphi(\mu) & (k=m+1 ; j=1, \ldots, m) \\
\zeta^{1-k} \varphi(\mu) \psi_{m-1}(\mu)^{-1} & (k=1, \ldots, m ; j=m+1) \\
0 & (k=j=m+1)\end{cases}
\end{aligned}
$$


where

$$
\begin{aligned}
& \eta_{2}(\mu):= \\
& \begin{cases}\frac{m(m-1)}{2}+\frac{\psi_{1}(\mu)}{\mu+1}\left\{m-1+(m+\varphi(\mu)) \sum_{l=1}^{m-1} \psi_{l}(\mu)^{-1}\right\} & (k=j) \\
\frac{m}{\zeta^{k-j}-1}+\frac{\psi_{1}(\mu)}{\mu+1}\left\{-1+(m+\varphi(\mu)) \sum_{l=1}^{m-1} \zeta^{(k-j) l} \psi_{l}(\mu)^{-1}\right\} & (k \neq j)\end{cases}
\end{aligned}
$$

Putting it into (3.1) and (3.2), we get

(3.6) $\frac{\partial f^{k}}{\partial q_{j}}\left(q^{0}, \mu\right)=-f(\mu)^{2} \psi(\mu)^{2} \zeta^{1-j}$

$$
\times\left\{\begin{array}{lr}
2(m-1+\varphi(\mu))-\frac{m-2+\varphi(\mu)}{2 m} \eta_{2}(\mu)-\eta_{1}(\mu) \quad(k, j=1, \ldots, m) \\
(2 m+1) \varphi(\mu) & (k=m+1 ; j=1, \ldots, m) \\
0 & (k=1, \ldots, m+1 ; j=m+1) .
\end{array}\right.
$$

In particular, we have

$$
\Gamma_{m+1}(\mu)=\left(f^{m+1}\right)^{-2} \frac{\partial^{2} \operatorname{det} A}{\partial q_{1} \partial q_{m+1}} \cdot\left(f^{m+1} \frac{\partial f^{k}}{\partial q_{j}}-f^{k} \frac{\partial f^{m+1}}{\partial q_{j}}\right)_{k, j=1, \ldots, m}
$$

at $\left(q^{0}, \mu\right)$.

Computation of $\left(\partial^{2} \operatorname{det} A / \partial q_{1} \partial q_{m+1}\right)\left(q^{0}, \mu\right)$. First we compute

$$
\begin{aligned}
& \frac{\partial^{2} \operatorname{det} A}{\partial q_{1} \partial q_{m+1}}\left(q^{0},-1\right) \\
& \quad=\operatorname{Tr}\left(\frac{\partial^{2} A}{\partial q_{1} \partial q_{m+1}}\left(q^{0},-1\right) \cdot B\left(q^{0},-1\right)+\frac{\partial A}{\partial q_{1}}\left(q^{0},-1\right) \cdot \frac{\partial B}{\partial q_{m+1}}\left(q^{0},-1\right)\right) .
\end{aligned}
$$

It is easy to see that,

$$
\frac{\partial^{2} \alpha_{k l}}{\partial q_{1} \partial q_{m+1}}\left(q^{0},-1\right)= \begin{cases}-2 & (k=1 ; l=m+1) \\ 2 & (k=m+1 ; l=1) \\ 0 & \text { elsewhere }\end{cases}
$$


On the other hand, we have

$$
\left.\frac{\partial}{\partial t}\right|_{t=0} Y_{t}(-1)=\left(\begin{array}{ccccc}
2-m & \zeta^{1} & \cdots & \zeta^{m-1} & 0 \\
1 & (2-m) \zeta^{1} & \cdots & \zeta^{m-1} & 0 \\
\vdots & \vdots & \ddots & \vdots & \vdots \\
1 & \zeta^{1} & \cdots & (2-m) \zeta^{m-1} & 0 \\
0 & 0 & \cdots & 0 & 0
\end{array}\right) .
$$

By putting these values into (3.3), we have

$$
\frac{\partial \beta_{k l}}{\partial q_{m+1}}\left(q^{0},-1\right)= \begin{cases}-(m-1) \zeta^{1-k}+\zeta^{1-l} & (k, l=1, \ldots, m) \\ (m-1) \zeta^{1-k} & (k=1, \ldots, m ; l=m+1) \\ -(m-1) \zeta^{1-l} & (k=m+1 ; l=1, \ldots, m) \\ 0 & (k=l=m+1) .\end{cases}
$$

Now, by a straightforward calculation, we have

$$
\frac{\partial^{2} \operatorname{det} A}{\partial q_{1} \partial q_{m+1}}\left(q^{0},-1\right)=m(m-1) \neq 0 .
$$

Since $\left(\partial^{2} \operatorname{det} A / \partial q_{1} \partial q_{m+1}\right)\left(q^{0}, \mu\right)$ is a polynomial in $\mu$, it does not vanish for any $\mu$ except for finite values.

Computation of the rank of $\Gamma_{m+1}(\mu)$. For any $\mu \in(0,1) \cup(1,+\infty)$ such that

$$
\frac{\partial^{2} \operatorname{det} A}{\partial q_{1} \partial q_{m+1}}\left(q^{0}, \mu\right) \neq 0
$$

define a cyclic matrix

$$
\Gamma_{m+1}^{0}:=-\frac{1}{(f \psi)^{2}}\left(\frac{\partial f^{k}}{\partial q_{j}}-\frac{f^{k}}{f^{m+1}} \frac{\partial f^{m+1}}{\partial q_{j}}\right)_{k, j=1, \ldots, m} \cdot C_{1},
$$

where $C_{1}=\operatorname{diag}\left[1, \zeta^{1}, \ldots, \zeta^{m-1}\right]$. Then it is clear that the rank of $\Gamma_{m+1}$ is equal to the rank of $\Gamma_{m+1}^{0}$. The $(k, j)$-component $\gamma_{k j}$ of $\Gamma_{m+1}^{0}$ is given by

$$
\gamma_{k j}=-\frac{m-1+\varphi}{m}-\frac{m-2+\varphi}{2 m} \eta_{2}-\eta_{1}
$$


and the eigenvalues $\chi_{1}, \ldots, \chi_{m}$ of $\Gamma_{m+1}^{0}$ are given by

$$
\begin{aligned}
& \chi_{l}(\mu)=\sum_{j=1}^{m} \gamma_{1 j}(\mu)\left(\zeta^{l}\right)^{j-1} \\
= & \begin{cases}-\frac{(\mu+1)\{(m-1) \mu+m+1\}(l-1)(l-m+1)}{4 \psi_{l}(\mu)} & (l=1, \ldots, m-1) \\
0 & (l=m) .\end{cases}
\end{aligned}
$$

Now it is clear that $\chi_{l}(\mu) \neq 0$ for $l=2, \ldots, m-2$, and $\Gamma_{m+1}^{0}$ is of rank $m-3$. Consequently, $\Gamma_{m+1}$ is of rank $m-3$ for any $\mu \in(0,1) \cup(1,+\infty)$ except for finite values.

Now, by Theorem 2.4, we get the following theorem:

Theorem 3.1. For almost all given unit vectors $v=\left\{v_{1}, \ldots, v_{n}\right\}(n \geq 5)$ in $\mathbf{R}^{3}$, and nonzero real numbers $a=\left\{a^{1}, \ldots, a^{n}\right\}$ satisfying $\sum_{j=1}^{n} a^{j} v_{j}=0$, there is a (non-branched) n-end catenoid $x: \mathbf{C} \backslash\left\{q_{1}, \ldots, q_{n}\right\} \rightarrow \mathbf{R}^{3}$ such that $\nu\left(q_{j}\right)=v_{j}$ and $a_{j}$ is the weight at the end $q_{j}$.

This theorem and the results for $n \leq 4$ ([L], [KUY1]) imply our main theorem in Introduction.

\section{Appendix A.}

In this appendix, we give two lemmas on real analytic families of algebraic equations which are applied in the proof of Proposition 1.7.

Lemma A.1. Let $\left\{f_{p}\left(q_{1}, \ldots, q_{n}\right)\right\}_{p \in \mathbf{R}^{l}}$ and $\left\{g_{p}\left(q_{1}, \ldots, q_{n}\right)\right\}_{p \in \mathbf{R}^{l}}$ be two real analytic families of polynomials on $\mathbf{C}$ of degree bounded by $m$. Suppose that there exists a non-empty open subset $U$ such that

$$
Z\left(f_{p}\right) \subset Z\left(g_{p}\right) \quad(p \in U) .
$$

Then (A.1) holds for all $p \in \mathbf{R}^{l}$ such that $f_{p} \not \equiv 0$.

Proof. For each $p \in \mathbf{R}^{l}$, since the degree of $f_{p}$ is bounded by $m, Z\left(f_{p}\right) \subset$ $Z\left(g_{p}\right)$ if and only if $\left(g_{p}\right)^{m}$ is divided by $f_{p}$. We operate a differential operator

$$
D^{\alpha}:=\frac{\partial^{|\alpha|}}{\partial q_{1}^{\alpha_{1}} \cdots \partial q_{n}^{\alpha_{n}}}
$$


into the rational function $\varphi_{p}:=\left(g_{p}\right)^{m} / f_{p}$. Let $\mathcal{N}^{\alpha}\left(\varphi_{p}\right)$ be a polynomial formally defined as

$$
\mathcal{N}^{\alpha}\left(\varphi_{p}\right):=\left(f_{p}\right)^{|\alpha|+1} \cdot D^{\alpha} \varphi
$$

which is the numerator part of $D^{\alpha} \varphi$.

Now we fix an element $p_{0} \in \mathbf{R}^{l}$ such that $f_{p} \not \equiv 0$, and choose an element $q_{0} \in \mathbf{C}^{n}$ such that $f_{p_{0}}\left(q_{0}\right) \neq 0$. Since $f_{p}$ is real analytic with respect to the parameter $p$, we can take a subdomain $V$ of $U$ such that $f_{p}\left(q_{0}\right) \neq 0$ for all $p \in V$, and $\varphi_{p}$ is a polynomial on $\mathbf{C}$ of degree bounded by $m^{2}$ for any $p \in V$.

Hence for any multi-index $|\alpha|>m^{2}$, we have $\mathcal{N}^{\alpha}\left(\varphi_{p}\right)\left(q_{0}\right)=0$ for $p \in V$. By the real analyticity with respect to the parameter $p$, we have $\mathcal{N}^{\alpha}\left(\varphi_{p_{0}}\right)\left(q_{0}\right)=0$ for $|\alpha|>m^{2}$. Since $f_{p_{0}}\left(q_{0}\right) \neq 0$, we get $D^{\alpha} \varphi\left(q_{0}\right)=0$ for $|\alpha|>m^{2}$. Thus $\varphi_{p_{0}}$ is also a polynomial on $\mathbf{C}$.

The following lemma is easily proved by using the Cauchy-Riemann equation.

Lemma A.2. Let $\mathcal{W}_{0}$ be a totally real subset of $\mathbf{P}^{n-1}$ defined by

$$
\mathcal{W}_{0}:=\left\{\left[a^{1}, \ldots, a^{n}\right] \in \mathbf{P}^{n-1} ; a^{j} \in \mathbf{R}(j=1, \ldots, n)\right\} .
$$

Let $h$ be a homogeneous polynomial on $\mathbf{C}$. If $h$ is identically zero on a non-empty open subset in $\mathcal{W}_{0}$, then $h \equiv 0$ on $\mathbf{P}^{n-1}$.

\section{Appendix B.}

Let $A$ be an $n \times n$ matrix. The cofactor matrix $B$ of $A$ is the matrix satisfying the identity $B A=A B=\operatorname{det} A \cdot I$. In this appendix, we give an identity which is useful to compute a differential of the cofactor matrix of a singular matrix.

Let $\Omega$ be a domain in $\mathbf{C}$ containing the origin, and $A(q): \Omega \rightarrow M(n, \mathbf{C})$ a smooth map into the set of all $n \times n$ matrices. Let $B(q)$ be the cofactor matrix of $A(q)$. We set $A:=A(0)$ and $B:=B(0)$. Suppose that

$$
\operatorname{det} A=\left.\frac{\partial}{\partial q}\right|_{q=0} \operatorname{det} A(q)=0 .
$$

Then the following lemma holds. 
Lemma B.1. Let $X$ be an $n \times n$ matrix such that $\operatorname{Tr}(X B) \neq 0$. Then the following identity holds:

$$
\begin{aligned}
\frac{\partial B}{\partial q}(0)=\frac{1}{\operatorname{Tr}(X B)} & \left\{\operatorname{Tr}\left(\left.\frac{\partial A}{\partial q}(0) \cdot \frac{\partial Y_{t}}{\partial t}\right|_{t=0}\right) \cdot B\right. \\
& \left.-\left.\frac{\partial Y_{t}}{\partial t}\right|_{t=0} \cdot \frac{\partial A}{\partial q}(0) \cdot B-\left.B \cdot \frac{\partial A}{\partial q}(0) \cdot \frac{\partial Y_{t}}{\partial t}\right|_{t=0}\right\}
\end{aligned}
$$

where $Y_{t}$ is the cofactor matrix of $A+t X$.

Proof. We set $A_{t}(q):=A(q)+t X$, and denote by $B_{t}(q)$ its cofactor matrix. We have the following Taylor expansions:

$$
\begin{aligned}
& A_{t}(q)=(A+t X)+q \frac{\partial A}{\partial q}(0)+o(q) \\
& B_{t}(q)=Y_{t}+q \frac{\partial B_{t}}{\partial q}(0)+o(q) .
\end{aligned}
$$

Since $A_{t}(q) B_{t}(q)=\operatorname{det} A_{t}(q) \cdot I$, we have by taking the first degree terms that

$$
\left.\frac{\partial}{\partial q}\right|_{q=0} \operatorname{det} A_{t}(q) \cdot I=\frac{\partial A}{\partial q}(0) \cdot Y_{t}+(A+t X) \cdot \frac{\partial B_{t}}{\partial q}(0)
$$

Since

$$
\left.\frac{\partial}{\partial t}\right|_{t=0} \operatorname{det}(A+t X)=\operatorname{Tr}(X B) \neq 0
$$

$A+t X$ is non-singular around $t=0$. Hence we have

$$
\begin{aligned}
\frac{\partial B_{t}}{\partial q}(0) & =(A+t X)^{-1}\left(\left.\frac{\partial}{\partial q}\right|_{q=0} \operatorname{det} A_{t}(q) \cdot I-\frac{\partial A}{\partial q}(0) \cdot Y_{t}\right) \\
& =\frac{1}{\operatorname{det}(A+t X)}\left\{\left.\frac{\partial}{\partial q}\right|_{q=0} \operatorname{det} A_{t}(q) \cdot Y_{t}-Y_{t} \cdot \frac{\partial A}{\partial q}(0) \cdot Y_{t}\right\}
\end{aligned}
$$

Apply de L'Hospital rule to the right-hand side of

$$
\frac{\partial B}{\partial q}(0)=\lim _{t \rightarrow 0} \frac{\partial B_{t}}{\partial q}(0)
$$

Then we get the equality (B.2). 


\section{References.}

[B] R. L. Bryant, Surfaces in conformal geometry, Proc. Simpo. Pure Math. 48 (1988), 227-240.

[GR] H. Grauert and R. Remmert, Coherent Analytic Sheaves, Grundl. der Math. vol. 265, Springer-Verlag (1984).

[JM] L. P. Jorge and W. H. Meeks III, The topology of complete minimal surfaces of finite total Gaussian curvature, Topology 22 (1983), 203-221.

[Kar] H. Karcher, Construction of minimal surfaces, Surveys in Geometry 1989/1990, University of Tokyo.

[Kat] S. Kato, Construction of $n$-end catenoids with prescribed flux, Kodai Math. J. 18 (1995), 86-98.

[KS] R. Kusner and N. Schmitt, The spinor representation of minimal surfaces, preprint.

[KUY1] S. Kato, M. Umehara and K. Yamada, An inverse problem of the flux for minimal surfaces, Indiana Univ. Math. J. 46 (1997), 529-559.

[KUY2] S. Kato, M. Umehara and K. Yamada, General existence of minimal surfaces with prescribed flux II, in "Topics in Complex Analysys, Differential Geometry and Mathematical Physics", Edited by S. Demiev and K. Sekigawa, World Scientific, 1997, pp. 116-135.

[L] F. J. Lopez, The classification of complete minimal surfaces with total curva-

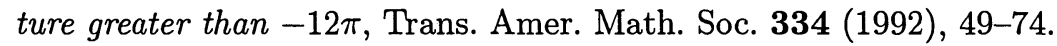

[LR] F. J. Lopez and A. Ros, On embedded complete minimal surfaces of genus zero, J. Differential Geometry 33 (1991), 293-300.

[M] W. H. Meeks III, The classification of complete minimal surfaces in $\mathbf{R}^{3}$ with

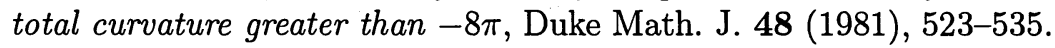

[RT] H. Rosenberg and E. Toubiana, Complete minimal surfaces and minimal herissons, J. Differential Geometry 28 (1988), 115-132.

[Ross1] W. Rossman, Minimal surfaces in $\mathbf{R}^{3}$ with dihedral symmetry, Tohoku Math. J. 47 (1995), 31-54.

[Ross2] W. Rossman, On embeddedness of area-minimizing disks, and an application to constructing complete minimal surfaces, preprint.

[UY] M. Umehara and K. Yamada, Surfaces of constant mean curvature $c$ in $H^{3}\left(-c^{2}\right)$ with prescribed hyperbolic Gauss map, Math. Ann. 304 (1996), 203224. 
[Xu] Y. Xu, Symmetric minimal surfaces in $\mathbf{R}^{3}$, Pacific J. Math. 171 (1995), 275296.

RECeIVEd May 30, 1997.

FACULTY OF SCIENCE, OSAKA CITY UNIVERSITY, OSAKA 558-8585 JAPAN

FACULTY OF SCIENCE HIROSHIMA UNIVERSITY, HigASHI-HIROSHIMA 739-8526 JAPAN

AND

FACULTY OF SCIENCE, KUMAMOTO UNIVERSITY, KUMAMOTO 860-8555 JAPAN

E-mail addresses: shinkato@sci.osaka-cu.ac.jp umehara@math.sci.hiroshima-u.ac.jp kotaro@gpo.kumamoto-u.ac.jp 